REPRESENTATION THEORY

An Electronic Journal of the American Mathematical Society

Volume 15, Pages 112-125 (February 7, 2011)

S 1088-4165(2011)00400-3

\title{
ON THE FOURIER INVERSION FORMULA FOR THE FULL MODULAR GROUP
}

\author{
KEITH R. OUELLETTE
}

\begin{abstract}
We offer a new proof of the Fourier inversion and Plancherel formulae for Maass-Eisenstein wave packets. The proof uses truncation, basic analysis, and classical Fourier theory. Brief sketches of the proofs due to Langlands, Lapid, and Casselman are then presented for comparison.
\end{abstract}

\section{INTRODUCTION}

The classical Fourier inversion formula states that one can recover a function $f \in C_{c}^{\infty}(\mathbb{R})$ from its Fourier transform $\hat{f}$ :

$$
f(x)=\frac{1}{2 \pi} \int_{-\infty}^{\infty} \hat{f}(s) e^{i s x} d s .
$$

Many other function spaces admit a Fourier theory. Let $\mathbb{H}$ be the upper halfplane and $\Gamma$ a discrete subgroup of $S L_{2}(\mathbb{R})$. The group $\Gamma$ acts by linear fractional transformations on $\mathbb{H}$. We consider such $\Gamma$ having one cusp and an orbit space $\Gamma \backslash \mathbb{H}$ with finite area. By replacing $\Gamma$ with a conjugate of $\Gamma$, we may assume that the cusp is at $i \infty$ and that $\mathbb{Z}$ can be identified with $\Gamma \cap N$ where $N$ is the subgroup of unipotent matrices of $S L_{2}(\mathbb{R})$. For example, $\Gamma$ could be the full modular group $S L_{2}(\mathbb{Z})$. The Fourier inversion formula

$$
\varphi_{\#}(t)=\int_{\Gamma \backslash \mathbb{H}} E_{\varphi}(x, y) E_{-t}(x, y) \frac{d x d y}{y^{2}}
$$

states that one can recover the function $\varphi \in C_{c}^{\infty}(i \mathbb{R})$ up to a projection $\varphi_{\#}$ by integrating its Maass-Eisenstein wave packet $E_{\varphi}$ against the Maass-Eisenstein series kernel $E_{-t}$.

The main goal of this paper is to offer a new proof of this formula and compare it to existing proofs. The new proof shows convergence of the integral for imaginary $t$, writes the integral as a sum of two integrals according to the orthogonal decomposition afforded by a truncation operator, and ultimately expresses each integral as a partial Mellin transform of the inverse Mellin transform of $\varphi_{\#}$. Our new proof generalizes nicely to cuspidal Eisenstein series defined on arbitrary adèlic reductive groups and will soon appear in one of our subsequent papers. We plan on removing the cuspidal restriction in another paper.

Received by the editors October 21, 2006 and, in revised form, December 10, 2010. 2010 Mathematics Subject Classification. Primary 22E45; Secondary 11 F72.

(C)2011 American Mathematical Society Reverts to public domain 28 years from publication 


\section{History}

The theory of using non-holomorphic Eisenstein series to construct solutions to certain partial differential equations first appears in the work of Hans Maass. In $[\underline{8}$, Maass introduced a real-analytic Eisenstein series $E_{s}$ for congruence subgroups $\Gamma$. Defining his series initially for an appropriate open right half-plane, Maass proved that $E_{s}$ meromorphically continues in $s$ to $\mathbb{C}$ due to the fact that $L$-functions occur in its Fourier expansion for such $\Gamma$. This meromorphic continuation of $E_{s}$ for values on the critical line $\operatorname{Re} s=\frac{1}{2}$ (or Re $s=0$ depending on how it is defined) satisfies such equations.

Walter Roelcke, a student of Maass, was inspired by Maass' work and considered $E_{s}$ for discrete arithmetic $\Gamma$ that were not congruence subgroups. In [10, Roelcke meromorphically continued such $E_{s}$ to all complex values of $s$ such that $s^{2}-\frac{1}{4}$ is not in the interval $(-\infty, 0]$ using the spectral theory of the Laplacian differential operator. Roelcke's Theorem 37 in ([10], p. 91) shows that his normalized meromorphically-continued Eisenstein series $E_{s}$ appears in the continuous spectrum of the negative Laplacian for values $s=1+2 i r$ where $r>0$.

Atle Selberg, in search of what he called a "trace formula", a generalization of the Poisson summation formula, was the first to meromorphically continue $E_{s}$ to the entire complex plane for discrete subgroups $\Gamma$ mentioned in the introduction. In fact, Selberg asserted in [12] that $E_{s}$ is regular for Re $s \geq \frac{1}{2}$ with possibly a finite number of simple poles in $\left(\frac{1}{2}, 1\right]$.

Circa 1965, R. P. Langlands provided details for establishing the meromorphic continuation of convergent Eisenstein series for any locally symmetric space of finite volume. Using residue calculus, he completely determined the continuous spectrum for such a space in terms of discrete spectra for spaces of smaller dimension. In [6], Langlands also established the Plancherel formula, which states an identity between an inner product of wave packets $E_{\varphi}$ and $E_{\psi}$ and an inner product of the functions $\varphi$ and $\psi$ used to build those wave packets. In the case of the continuous spectrum $L_{\text {cont }}^{2}(\Gamma \backslash \mathbb{H})$, the Plancherel formula can be written:

$$
\left\langle E_{\varphi}, E_{\psi}\right\rangle_{\Gamma \backslash \mathbb{H}}=\frac{1}{2}\left\langle\varphi_{\#}, \psi_{\#}\right\rangle_{i \mathbb{R}} .
$$

We see that $\varphi$ and $\psi$ become projections in the inner product on the right. Moeglin and Waldspurger carried Langlands' techniques over to adèlic reductive groups in [9].

In 1999, W. Casselman, assuming meromorphic continuation of $E_{s}$, discovered another proof of the Plancherel formula by proving the Fourier inversion formula for $L_{\text {cont }}^{2}(\Gamma \backslash \mathbb{H})$. His proof in 3 used a very interesting distribution on the space of smooth compactly-supported functions on the imaginary axis:

$$
\left(P V \frac{T^{-s}}{-s}\right)[\varphi]=\lim _{\epsilon \rightarrow 0} \frac{1}{2 \pi i} \int_{\substack{i \mathbb{R} \\|s| \geq \epsilon}} \varphi(s) \frac{T^{-s}}{-s} d s .
$$

More importantly, however, it circumvented an argument found in [5] and in [6] that involved a contour shift from a line where the Eisenstein series converges to where the inner product of the wave packet and the Eisenstein series converges. Although this contour shift from a line in the region $\operatorname{Re} s>1$ to the line $\operatorname{Re} s=0$ was a very simple procedure in the classical case, its complexity increased considerably 
when generalizing the argument to groups of higher rank. While attempting to generalize Casselman's argument to arbitrary adèlic reductive groups, we discovered an alternative proof that also avoided the complicated contour shifts as well as Casselman's distribution.

In [7, Erez Lapid recently established another proof of the Plancherel formula for wave packets built from discrete (not necessarily cuspidal) Eisenstein series in the reductive adèlic group setting. His proof uses a formula for the inner product of two truncated discrete Eisenstein series.

\section{BACKGROUND}

We now explain some basic terminology and definitions used in the proof.

\subsection{Mellin transforms.}

Definition 3.1. Let $\varphi$ be a smooth compactly-supported function on $i \mathbb{R}$. We define the shifted inverse Mellin transform of $\varphi$ to be the function $\check{\varphi}: \mathbb{R}^{+} \rightarrow \mathbb{C}$ given by

$$
\check{\varphi}(y)=y^{\frac{1}{2}}\left[\frac{1}{2 \pi i} \int_{i \mathbb{R}} \varphi(s) y^{s} d s\right] .
$$

Remark 3.2. The choice of the kernel $y^{s}$ instead of the classical inverse Mellin transform kernel $y^{-s}$ is merely for simplicity.

The following identity

$$
\frac{1}{2 \pi i} \int_{i \mathbb{R}} \varphi(s) y^{s} d s=\frac{1}{2 \pi} \int_{-\infty}^{\infty} \varphi(i \cdot \operatorname{Im} s) e^{i(\operatorname{Im} s) \log y} d(\operatorname{Im} s)
$$

shows us that $y^{-\frac{1}{2}} \check{\varphi}(y)$ is the classical inverse Fourier transform of the $C_{c}^{\infty}(\mathbb{R})$ function $t \rightarrow \varphi(i t)$ evaluated at $\log y$. Therefore, it is natural to consider the following space of functions:

\section{Definition 3.3.}

$$
\mathcal{S}^{\frac{1}{2}}\left(\mathbb{R}^{+}\right)=\left\{y^{\frac{1}{2}} f(\log y): f \in \mathcal{S}(\mathbb{R})\right\}
$$

Notice that $\check{\varphi}(y) \in \mathcal{S}^{\frac{1}{2}}\left(\mathbb{R}^{+}\right)$. Thus $\mathcal{S}^{\frac{1}{2}}\left(\mathbb{R}^{+}\right)$is a space formed by composing Schwartz functions $f$ on $\mathbb{R}$ with $\log y$ (where $y>0$ ) and then multiplying the resulting function by $y^{\frac{1}{2}}$.

Definition 3.4. Let $\Phi \in \mathcal{S}^{\frac{1}{2}}\left(\mathbb{R}^{+}\right)$. We define the Mellin transform of $\Phi$ to be the function $\hat{\Phi}: i \mathbb{R} \rightarrow \mathbb{C}$ given by

$$
\hat{\Phi}(s)=\int_{0}^{\infty}\left[y^{-\frac{1}{2}} \Phi(y)\right] y^{-s} \frac{d y}{y} .
$$

Notice that we multiply $\Phi$ by the factor $y^{-\frac{1}{2}}$ before applying the Mellin transform with kernel $y^{-s}$. For any smooth compactly-supported function $\varphi$ on $i \mathbb{R}$, the classical theory still gives us $\hat{\varphi}=\varphi$.

3.1.1. Partial Mellin transforms. The following lemma and its corollary, although simple results from basic analysis, are fundamental to the new proof of the Fourier inversion formula and separate it from the proofs of Casselman, Langlands, and Lapid. 
Lemma 3.5. Let $T \geq 1$ and $f$ be a function such that for every $N \in \mathbb{N}$, there exists a $C_{N}>0$ such that

$$
\sup _{y \in \mathbb{R}^{+}}(1+|\log y|)^{N}|f(y)| \leq C_{N}<\infty .
$$

Then the following integrals converge absolutely and uniformly in vertical strips for Re $s \leq 0$ :

$$
\begin{aligned}
& \int_{T}^{\infty} f(y) y^{s} \frac{d y}{y} \\
& \int_{0}^{T} f(y) y^{-s} \frac{d y}{y} .
\end{aligned}
$$

Proof. Let $\sigma=\operatorname{Re} s \leq 0$ and $N>1$. Note that $\left|y^{s}\right|=y^{\sigma} \leq 1$ on $[T, \infty)$ and $\left|y^{-s}\right|=y^{-\sigma} \leq 1$ on $[0,1]$. Thus

$$
\begin{aligned}
& \int_{T}^{\infty}\left|f(y) y^{s}\right| \frac{d y}{y} \leq C_{N} \int_{T}^{\infty}(1+\log y)^{-N} \frac{d y}{y}=C_{N} \int_{1+\log T}^{\infty} u^{-N} d u<\infty \\
& \int_{0}^{1}\left|f(y) y^{-s}\right| \frac{d y}{y} \leq C_{N} \int_{0}^{1}(1-\log y)^{-N} \frac{d y}{y}=C_{N} \int_{1}^{\infty} u^{-N} d u<\infty
\end{aligned}
$$

Finally,

$$
\int_{1}^{T}\left|f(y) y^{-s}\right| \frac{d y}{y} \leq \int_{1}^{T}|f(y)| y^{-1-\sigma} d y \ll \int_{1}^{T}|f(y)| d y<\infty
$$

since $y^{-1-\sigma}$ is bounded uniformly on a given vertical strip.

Corollary 3.6. The integrals of Proposition 3.5 are holomorphic in s for Re $s<0$ and continuous for Re $s \leq 0$.

Proof. By uniform convergence, we may differentiate under the integral sign for Re $s<0$. If we let $s=\sigma+i \tau$,

$$
\begin{aligned}
& \lim _{\sigma \rightarrow 0} \int_{T}^{\infty} f(y) y^{s} \frac{d y}{y}=\int_{T}^{\infty} f(y) y^{i \tau} \frac{d y}{y}, \\
& \lim _{\sigma \rightarrow 0} \int_{0}^{T} f(y) y^{-s} \frac{d y}{y}=\int_{0}^{T} f(y) y^{-i \tau} \frac{d y}{y} .
\end{aligned}
$$

Let $\epsilon>0$. We can choose $\delta>0$ so that $\left|y^{\sigma}-1\right|<\epsilon$ and $\left|y^{-\sigma}-1\right|<\epsilon$ when $0 \leq \sigma<\delta$. Thus

$$
\begin{aligned}
& \left|\int_{T}^{\infty} f(y) y^{s} \frac{d y}{y}-\int_{T}^{\infty} f(y) y^{i \tau} \frac{d y}{y}\right| \leq \int_{T}^{\infty}|f(y)|\left|y^{\sigma}-1\right| \frac{d y}{y}<\epsilon \int_{T}^{\infty}|f(y)| \frac{d y}{y} \\
& \left|\int_{0}^{T} f(y) y^{-s} \frac{d y}{y}-\int_{0}^{T} f(y) y^{-i \tau} \frac{d y}{y}\right| \leq \int_{0}^{T}|f(y)|\left|y^{-\sigma}-1\right| \frac{d y}{y}<\epsilon \int_{0}^{T}|f(y)| \frac{d y}{y} .
\end{aligned}
$$

Since $\epsilon>0$ was arbitrary, the result follows. 
3.2. Eisenstein series. To get the appropriate Fourier transform kernel for $C_{c}^{\infty}(\Gamma \backslash \mathbb{H})$, we define the Maass-Eisenstein series, mention some of its properties, and define a more general Eisenstein series. For any function $F$ on $\mathbb{H}$, we make the identification $F(z)=F(x, y)$ where $z=x+i y$. It is customary to identify $z$ with a unique coset of $S L(2, \mathbb{R}) / S O(2, \mathbb{R})$. Let $G=S L_{2}(\mathbb{R})$ and define the following subgroups:

$$
\begin{aligned}
& N=\left\{\left(\begin{array}{cc}
1 & x \\
0 & 1
\end{array}\right): x \in \mathbb{R}\right\}, \\
& A=\left\{\left(\begin{array}{cc}
y^{\frac{1}{2}} & 0 \\
0 & y^{-\frac{1}{2}}
\end{array}\right): y \in \mathbb{R}^{+}\right\}, \\
& K=S O_{2}(\mathbb{R}) .
\end{aligned}
$$

Every $g \in G$ can be uniquely represented as $g=n a k$ where $n \in N, a \in A$, and $k \in K$. Let $P=N A$.

Definition 3.7. For $z \in \mathbb{H}$, we define the Maass-Eisenstein series

$$
E_{s}(z)=\sum_{\gamma \in P \cap \Gamma \backslash \Gamma}(\operatorname{Im} \gamma z)^{\frac{1}{2}+s}
$$

for $\operatorname{Re} s>\frac{1}{2}$, where it converges absolutely and uniformly on compact sets.

Selberg showed that it extends to a meromorphic function on $\mathbb{C}$ which is smooth on $i \mathbb{R}$. We will use $E_{s}$ to also denote the meromorphic continuation. It is an eigenfunction of the hyperbolic Laplacian

and satisfies

$$
\Delta=y^{2}\left(\frac{\partial^{2}}{\partial x^{2}}+\frac{\partial^{2}}{\partial y^{2}}\right)
$$

$$
\Delta E_{s}=\left(s+\frac{1}{2}\right)\left(s-\frac{1}{2}\right) E_{s} .
$$

In fact, the continuous spectrum of the hyperbolic Laplacian on $L^{2}(\Gamma \backslash \mathbb{H})$ is obtained by integrating smooth compactly-supported functions $\varphi(s)$ on $i \mathbb{R}$ against $E_{s}$. Since the Eisenstein series is invariant under the translation

$$
\left(\begin{array}{ll}
1 & 1 \\
0 & 1
\end{array}\right) z=z+1
$$

for $z \in \mathbb{H}$, it is periodic in $x$ with period 1. Therefore, it has a Fourier series expansion

$$
E_{s}(z)=\sum_{n \in \mathbb{Z}} a_{n}(y) e^{2 \pi i n x}
$$

where the Fourier coefficients are functions of $y$. The constant term $a_{0}(y)$ of this expansion is given by

$$
\left(E_{s}\right)_{0}(y)=y^{\frac{1}{2}+s}+c(s) y^{\frac{1}{2}-s}
$$

for all $s$ where the meromorphic continuation $E_{s}$ is defined. The function $c(s)$ appearing in the constant term is called the Harish-Chandra $c$-function. When $s \in i \mathbb{R}$, it satisfies

$$
|c(s)|^{2}=c(s) c(-s)=1 .
$$

The Eisenstein series satisfies the functional equation

$$
E_{s}=c(s) E_{-s} .
$$


In the case where $\Gamma=S L_{2}(\mathbb{Z})$, the Harish-Chandra $c$-function is given explicitly by

$$
c(s)=\frac{\xi(2 s)}{\xi(1+2 s)}
$$

where $\xi(s)=\pi^{-\frac{s}{2}} \Gamma\left(\frac{s}{2}\right) \zeta(s)$ is the completed Riemann zeta-function.

Definition 3.8. For $\Phi$ on $N(P \cap \Gamma) \backslash \mathbb{H}$, define

$$
E_{P}^{G}(\Phi)(z)=\sum_{\gamma \in P \cap \Gamma \backslash \Gamma} \Phi(\gamma z)
$$

We call this the general Eisenstein series attached to $\Phi$.

If $\Phi_{s}(z)=(\operatorname{Im} z)^{\frac{1}{2}+s}$, we get the familiar Maass-Eisenstein series.

\subsection{Fourier-Eisenstein transform and wave packets.}

Definition 3.9. If $F \in C_{c}^{\infty}(\Gamma \backslash \mathbb{H})$, define the Fourier-Eisenstein transform:

$$
\hat{F}(s)=\int_{\Gamma \backslash \mathbb{H}} F(x, y) E_{-s}(x, y) \frac{d x d y}{y^{2}} .
$$

A natural question to ask is whether one can recover $F$ from $\hat{F}$. Selberg tells us that

$$
F(x, y)-\frac{1}{2 \pi i} \int_{i \mathbb{R}} \hat{F}(s) E_{s}(x, y) d s
$$

is the sum of a cusp form (which has a vanishing constant term) plus a constant. Hence (3.1) is a square-integrable function that lies in the discrete spectrum. Therefore, we shall instead start with $\varphi \in C_{c}^{\infty}(i \mathbb{R})$, define the wave packet, and explore some of its properties to see if we can eventually recover $\varphi$ from it.

Definition 3.10. Let $\varphi \in C_{c}^{\infty}(i \mathbb{R})$. Then the wave packet $E_{\varphi}$ is defined as

$$
E_{\varphi}(z)=\frac{1}{2 \pi i} \int_{i \mathbb{R}} \varphi(s) E_{s}(z) d s .
$$

Observe that the periodicity of the Eisenstein series gives us the periodicity of the wave packet:

$$
\begin{aligned}
E_{\varphi}(z+1) & =\frac{1}{2 \pi i} \int_{i \mathbb{R}} \varphi(s) E_{s}(z+1) d s \\
& =\frac{1}{2 \pi i} \int_{i \mathbb{R}} \varphi(s) E_{s}(z) d s \\
& =E_{\varphi}(z) .
\end{aligned}
$$

Lemma 3.11. The constant term of the wave packet $E_{\varphi}$ is $y^{\frac{1}{2}}$ multiplied by the inverse Mellin transform of $\varphi_{\#}$ where

$$
\varphi_{\#}(s)=\varphi(s)+c(-s) \varphi(-s) .
$$

Proof. We can use the constant term of the Eisenstein Series $E_{s}$ to get the constant term of the wave packet $E_{\varphi}$ :

$$
\begin{aligned}
\left(E_{\varphi}\right)_{0}(z) & =\frac{1}{2 \pi i} \int_{i \mathbb{R}} \varphi(s)\left(E_{s}\right)_{0}(z) d s \\
& =\frac{1}{2 \pi i} \int_{i \mathbb{R}} \varphi(s) y^{\frac{1}{2}+s} d s+\frac{1}{2 \pi i} \int_{i \mathbb{R}} \varphi(s) c(s) y^{\frac{1}{2}-s} d s .
\end{aligned}
$$


Making a substitution $s \rightarrow-s$ in the second integral above, we get

$$
\left(E_{\varphi}\right)_{0}(z)=\frac{1}{2 \pi i} \int_{i \mathbb{R}} \varphi(s) y^{\frac{1}{2}+s} d s+\frac{1}{2 \pi i} \int_{i \mathbb{R}} \varphi(-s) c(-s) y^{\frac{1}{2}+s} d s .
$$

Thus, the constant term of $E_{\varphi}$ is

$$
\left(E_{\varphi}\right)_{0}(z)=\frac{1}{2 \pi i} \int_{i \mathbb{R}}[\varphi(s)+c(-s) \varphi(-s)] y^{\frac{1}{2}+s} d s=y^{\frac{1}{2}}\left[\frac{1}{2 \pi i} \int_{i \mathbb{R}} \varphi_{\#}(s) y^{s} d s\right] .
$$

Remark 3.12. Note that $\varphi_{\#}$ is twice the projection of $\varphi$ onto the space of functions satisfying

$$
\varphi_{\#}(-s)=c(s) \varphi_{\#}(s)
$$

Proof.

$$
\begin{aligned}
\varphi_{\#}(-s) & =\varphi(-s)+c(s) \varphi(s) \\
& =c(s) c(-s) \varphi(-s)+c(s) \varphi(s) \\
& =c(s) \varphi_{\#}(s) .
\end{aligned}
$$

3.4. Asymptotics and convergence of the inner product. In order for the Fourier inversion formula to make sense, we must show that the Petersson inner product

$$
\left\langle E_{\varphi}, E_{t}\right\rangle_{\Gamma \backslash \mathbb{H}}=\int_{\Gamma \backslash \mathbb{H}} E_{\varphi}(x, y) \overline{E_{t}(x, y)} \frac{d x d y}{y^{2}}
$$

converges absolutely for $t \in i \mathbb{R}$. This will imply square-integrability of the wave packet. To this end, we recall the definition of a Siegel set and some basic growth conditions for functions on $\Gamma \backslash \mathbb{H}$.

Definition 3.13. Let $r>0$ and $T>0$. We call the following a standard Siegel set.

$$
\mathfrak{S}_{r, T}=\{z=x+i y:|x|<r, y \geq T\} .
$$

For $T$ large enough and appropriate choice of $r$, there is a fundamental domain $\mathcal{F}$ for $\Gamma \backslash \mathbb{H}$ such that $\mathcal{F}=\mathfrak{S}_{r, T} \cup C$ where $C$ is compact.

For the following definitions, let $F: \Gamma \backslash \mathbb{H} \rightarrow \mathbb{C}$ be a smooth function.

Definition 3.14. $F$ is of uniform moderate growth on a standard Siegel set $\mathfrak{S}$ if there exists a real number $N$ such that

$$
|X F(z)| \ll_{X} y^{N}
$$

for all $z \in \mathfrak{S}$ and for all $X$ in the universal enveloping algebra of $\mathfrak{g}$, the Lie algebra of $S L_{2}(\mathbb{R})$. In other words, $F$ and all of its derivatives satisfy an asymptotic estimate of some uniform power of $y$ on $\mathfrak{S}$.

Definition 3.15. $F$ is tempered on $\mathfrak{S}$ if for some $N>1$, we have

$$
|F(z)| \ll y^{\frac{1}{2}} \log ^{-N} y .
$$

Definition 3.16. $F$ is rapidly decreasing on $\mathfrak{S}$ if for all real $N$, we have

$$
|F(z)| \ll y^{-N} \text {. }
$$

The following lemma shows that the Maass-Eisenstein series is essentially asymptotic to its constant term, explaining why it is not square-integrable. 
Lemma 3.17. Let $N \in \mathbb{N}$ where $N \geq 2$. For sufficiently large $y>0$, the MaassEisenstein series $E_{s}(z)$ satisfies the growth condition

$$
\left|E_{s}(z)-\left(y^{\frac{1}{2}+s}+c(s) y^{\frac{1}{2}-s}\right)\right| \ll y^{-N}
$$

uniformly in s on compact subsets of $i \mathbb{R}$.

Proof. By Theorem 11.9 in ([2], p. 108), the meromorphic continuation of $E_{s}(z)$ in $s$ to $\mathbb{C}$ is an automorphic form for $\Gamma$. By Lemma 7.4 in ([2, p. 71), if $\left\{X_{1}, X_{2}, X_{3}\right\}$ is a basis of the Lie algebra $\mathfrak{g}$, there exists a constant $c>0$ independent of $E_{s}$ such that

$$
\left|E_{s}(z)-\left(y^{\frac{1}{2}+s}+c(s) y^{\frac{1}{2}-s}\right)\right| \leq c\left(\sum_{i=1}^{3}\left|X_{i} E_{s}\right|_{0}\right) y^{-\alpha}
$$

since the constant term of $E_{s}(z)$ is $y^{\frac{1}{2}+s}+c(s) y^{\frac{1}{2}-s}$. The constant terms $\left|X_{i} E_{s}\right|_{0}$ are continuous in $s$. Hence, they are bounded if $s$ ranges over a compact subset $\Omega$ of $i \mathbb{R}$. Therefore, there exists $\alpha \neq 0$ and $\widetilde{c}>0$ independent of $s$ (but depending on $\Omega$ ) such that

$$
\left|E_{s}(z)-\left(y^{\frac{1}{2}+s}+c(s) y^{\frac{1}{2}-s}\right)\right| \leq \widetilde{c} y^{-\alpha}
$$

for all $s \in \Omega$. In other words, the constant in the uniform moderate growth estimate for the automorphic form $E_{s}$ can be chosen uniformly on $\Omega$. We now follow the arguments in Theorem 7.5 and Corollary 7.6(ii) in ([2, p. 73) to conclude that not only is $E_{s}(z)-\left(y^{\frac{1}{2}+s}+c(s) y^{\frac{1}{2}-s}\right)$ rapidly decreasing on a standard Siegel set $\mathfrak{S}_{r, T}$ but also that the constant which appears can be chosen uniformly on $\Omega$. In particular, for all $N \in \mathbb{N}$ where $N>1$, we have that

$$
\left|E_{s}(z)-\left(y^{\frac{1}{2}+s}+c(s) y^{\frac{1}{2}-s}\right)\right| \ll y^{-N}
$$

uniformly in $s$ on the compact subset $\Omega$ of $i \mathbb{R}$.

Theorem 3.18. Let $t \in i \mathbb{R}$. Then $\left\langle E_{\varphi}, E_{t}\right\rangle_{\Gamma \backslash \mathbb{H}}$ converges absolutely.

Proof. It suffices to show absolute convergence on a standard Siegel set $\mathfrak{S}_{r, T}$. Let $\Omega$ be the support of $\varphi_{\#}$ which is a smooth compactly-supported function on $i \mathbb{R}$. Recall $\varphi(s)$ and $c(s)$ are smooth on $i \mathbb{R}$ and $\varphi(s)$ is compactly supported on $i \mathbb{R}$. Since the Fourier transform of a Schwartz function is Schwartz,

$$
\left(E_{\varphi}\right)_{0}(z)=y^{\frac{1}{2}}\left[\frac{1}{2 \pi i} \int_{i \mathbb{R}} \varphi_{\#}(s) e^{s \log y} d s\right]=O\left(y^{\frac{1}{2}} \log ^{-N} y\right)
$$

for any $N>1$. By Lemma 3.17 .

$$
\left|\left(E_{t}\right)_{0}(z)\right| \ll y^{\frac{1}{2}}
$$

where the constant in the bound only depends on $\Omega$, not $t$. Thus, for large $T>0$,

$$
\begin{aligned}
\int_{\mathfrak{S}_{r, T}}\left|E_{\varphi}(x, y)\right|\left|E_{t}(x, y)\right| \frac{d x d y}{y^{2}} & =\int_{T}^{\infty} \int_{-r}^{r}\left|E_{\varphi}(x, y)\right|\left|E_{t}(x, y)\right| d x \frac{d y}{y^{2}} \\
& \ll \int_{T}^{\infty} \log ^{-N} y \frac{d y}{y} \\
& =\int_{\log T}^{\infty} u^{-N} d u<\infty
\end{aligned}
$$

which gives the result.

Corollary 3.19. $E_{\varphi} \in L^{2}(\Gamma \backslash \mathbb{H})$. 
Proof. Let $\Omega$ be the compact support of $\varphi(s)$ :

$$
\begin{aligned}
\left|\left\langle E_{\varphi}, E_{\varphi}\right\rangle_{\Gamma \backslash \mathbb{H}}\right| & \leq \frac{1}{2 \pi} \int_{\Omega}|\varphi(s)|\left|\left\langle E_{\varphi}, E_{s}\right\rangle\right||d s| \\
& \leq \frac{1}{2 \pi} \max _{s \in \Omega}\left|\varphi(s)\left\langle E_{\varphi}, E_{s}\right\rangle\right| \int_{\Omega}|d s| \\
& <\infty .
\end{aligned}
$$

3.5. Adjoint relation. A key ingredient in the new proof of the Fourier inversion theorem is the adjoint relation which is stated and proved in ([2, p. 97).

Lemma 3.20. Let $F: \Gamma \backslash \mathbb{H} \rightarrow \mathbb{C}$ have constant term $F_{0}: N(P \cap \Gamma) \backslash \mathbb{H} \rightarrow \mathbb{C}$, then

$$
F_{0}(z)=\int_{N \cap \Gamma \backslash N} F(n z) d n
$$

Let $E_{P}^{G}(\Phi)$ be the general Eisenstein Series attached to $\Phi: N(P \cap \Gamma) \backslash \mathbb{H} \rightarrow \mathbb{C}$. Then the following identity formally holds:

$$
\left\langle F, E_{P}^{G}(\Phi)\right\rangle_{\Gamma \backslash \mathbb{H}}=\left\langle F_{0}, \Phi\right\rangle_{N \backslash \mathbb{H}} .
$$

Proof. The left $\Gamma$-invariance of $F$ gives us

$$
\int_{\Gamma \backslash \mathbb{H}} F(z) \sum_{\gamma \in(P \cap \Gamma) \backslash \Gamma} \Phi(\gamma z) \frac{d x d y}{y^{2}}=\int_{\Gamma \backslash \mathbb{H}} \sum_{(P \cap \Gamma) \backslash \Gamma} F(\gamma z) \Phi(\gamma z) \frac{d x d y}{y^{2}} .
$$

We combine integrals to get

$$
\int_{\Gamma \backslash \mathbb{H}} \sum_{(P \cap \Gamma) \backslash \Gamma} F(\gamma z) \Phi(\gamma z) \frac{d x d y}{y^{2}}=\int_{P \cap \Gamma \backslash \mathbb{H}} F(z) \Phi(z) \frac{d x d y}{y^{2}} .
$$

Now we express the integral as an iterated integral:

$$
\int_{P \cap \Gamma \backslash \mathbb{H}} F(z) \Phi(z) \frac{d x d y}{y^{2}}=\int_{(P \cap \Gamma) N \backslash \mathbb{H} P \cap \Gamma \backslash(P \cap \Gamma) N} \int_{P(n z) \Phi(n z) d n \frac{d x d y}{y^{2}} .}
$$

The left $(P \cap \Gamma) N$-invariance of $\Phi$ gives us

$$
\int_{(P \cap \Gamma) N \backslash \mathbb{H} P} \int_{P \cap \Gamma \backslash(P \cap \Gamma) N} F(n z) d n \Phi(z) \frac{d x d y}{y^{2}} .
$$

Borel shows that

$$
\int_{P \cap \Gamma \backslash(P \cap \Gamma) N} F(n z) d n=2 F_{0}(z) .
$$

Since $N \backslash \mathbb{H}$ is a 2 -fold cover of $(P \cap \Gamma) N \backslash \mathbb{H}$,

$$
\left\langle 2 F_{0}, \Phi\right\rangle_{(P \cap \Gamma) N \backslash \mathbb{H}}=\left\langle F_{0}, \Phi\right\rangle_{N \backslash \mathbb{H}} .
$$


3.6. Truncation and orthogonal decomposition. A problem in trying to use the adjoint relation to prove the Fourier inversion theorem is the inability to express $E_{-s}$ as an Eisenstein series $E_{P}^{G}\left(\Phi_{s}\right)$ for such $s$. Truncation allows us a way to represent $E_{-s}$ as a sum of mutually orthogonal square-integrable Eisenstein series so that we can use the adjoint relation.

Definition 3.21. Let $T>0$. We define the truncation $\Lambda_{G}^{P} F$ of a function $F$ on $\Gamma \backslash \mathbb{H}$ to be

$$
\Lambda_{G}^{P} F(z)=\sum_{\gamma \in P \cap \Gamma \backslash \Gamma} \chi_{(T, \infty)} F_{0}(\operatorname{Im} \gamma z)=E_{P}^{G}\left(\chi_{(T, \infty)} F_{0}\right)
$$

where $\chi_{(T, \infty)}$ is the characteristic function of $(T, \infty)$.

We define the truncation $\Lambda_{G}^{G} F$ to be

$$
\Lambda_{G}^{G} F(z)=F(z)-\Lambda_{G}^{P} F(z) .
$$

Proposition 3.22. For any $T>1$, the following decomposition is orthogonal:

$$
F=\Lambda_{G}^{G} F+\Lambda_{G}^{P} F .
$$

Proof. We reproduce the proof in [3. Let $\mathcal{H}_{\mathcal{T}}=\{z \in \mathbb{H}: \operatorname{Im} z \geq T\}$. Let $\gamma \in \Gamma$. If $\gamma \in \Gamma \cap P$, then $\gamma$ maps $\mathcal{H}_{\mathcal{T}}$ conformally onto itself. If $\gamma \notin P$, then $\gamma$ maps $\mathcal{H}_{\mathcal{T}}$ conformally onto a disk in the region where $\operatorname{Im} z<1$. Thus $T>1$ ensures orthogonality of the decomposition.

Remark 3.23. $\Lambda_{G}^{G} E_{P}^{G}(\Phi)(z)$ is the general Eisenstein series attached to the function

$$
\Phi-\chi_{(T, \infty)}\left(E_{P}^{G} \Phi\right)_{0} .
$$

Notice that

$$
\Lambda_{G}^{G} E_{P}^{G}(\Phi)=E_{P}^{G}(\Phi)-\Lambda_{G}^{P} E_{P}^{G}(\Phi)=E_{P}^{G}(\Phi)-E_{P}^{G}\left(\chi_{(T, \infty)}\left(E_{P}^{G} \Phi\right)_{0}\right) .
$$

For $T>1$ we get an orthogonal decomposition for $E_{-s}$,

$$
E_{-s}=\Lambda_{G}^{G} E_{-s}+\Lambda_{G}^{P} E_{-s}
$$

for any $s$ where the meromorphic continuation of $E_{-s}$ is defined.

\section{A NEW PROOF OF THE FOURIER INVERSION FORMULA}

In this section, we will be using the following (non-Hermitian) bilinear forms,

$$
\langle f, g\rangle_{X}=\int_{X} f(x) g(x) d x
$$

and will show that for $t \in i \mathbb{R}$,

$$
\left\langle E_{\varphi}, E_{-t}\right\rangle_{\Gamma \backslash \mathbb{H}}=\varphi_{\#}(t) .
$$

New proof of the Fourier inversion formula. Let $T>1$. For $\operatorname{Re} t<-\frac{1}{2}$, we can use the series definition of $E_{-t}$ to write the truncated Maass-Eisenstein series as a general Eisenstein series:

$$
\Lambda_{G}^{G} E_{-t}(z)=\sum_{\Gamma \cap P \backslash \Gamma} \operatorname{Im}(\gamma z)^{\left(\frac{1}{2}-t\right)}\left(1-\widetilde{\chi_{(T, \infty)}}\right)(\gamma z)-c(-t) \operatorname{Im}(\gamma z)^{\left(\frac{1}{2}+t\right)} \widetilde{\chi_{(T, \infty)}}(\gamma z)
$$


where $\widetilde{\chi_{(T, \infty)}}(z)=\chi_{(T, \infty)}(\operatorname{Im} z)$. By the adjoint relation, Lemma 3.20, we get for $\operatorname{Re} t<-\frac{1}{2}$,

$$
\left\langle E_{\varphi}, \Lambda_{G}^{G} E_{-t}\right\rangle_{\Gamma \backslash \mathbb{H}}=\int_{0}^{\infty} y^{\frac{1}{2}} \check{\varphi_{\#}}(y)\left[y^{-t}\left(1-\chi_{(T, \infty)}\right)(y)-c(-t) y^{t} \chi_{(T, \infty)}(y)\right] \frac{d y}{y^{2}}
$$

The right-hand side of (4.2) can also be written as

$$
\int_{0}^{T} y^{-\frac{1}{2}} \check{\varphi_{\#}}(y) y^{-t} \frac{d y}{y}-c(-t) \int_{T}^{\infty} y^{-\frac{1}{2}} \check{\varphi_{\#}}(y) y^{t} \frac{d y}{y} \text {. }
$$

The integrals

$$
\begin{aligned}
& \int_{0}^{T} y^{-\frac{1}{2}} \check{\varphi_{\#}}(y) y^{-t} \frac{d y}{y} \\
& \int_{T}^{\infty} y^{-\frac{1}{2}} \check{\varphi_{\#}}(y) y^{t} \frac{d y}{y}
\end{aligned}
$$

in (4.3) are holomorphic for $\operatorname{Re} t<-\frac{1}{2}$. Since $\check{\varphi_{\#}}(y) \in \mathcal{S}^{\frac{1}{2}}\left(\mathbb{R}^{+}\right)$, these integrals are even holomorphic for Re $t<0$ and continuous for Re $t \leq 0$ by Corollary 3.6 . The left-hand side of (4.2) is known to be meromorphic in $t$ and holomorphic for Re $t=0$. Notice (4.3) is meromorphic for Re $t \leq 0$ and continuous for $\operatorname{Re} t=0$. Therefore, since both sides of (4.2) are defined for Re $t=0$, the formula

$$
\left\langle E_{\varphi}, \Lambda_{G}^{G} E_{-t}\right\rangle_{\Gamma \backslash \mathbb{H}}=\int_{0}^{T} y^{-\frac{1}{2}} \check{\varphi_{\#}}(y) y^{-t} \frac{d y}{y}-c(-t) \int_{T}^{\infty} y^{-\frac{1}{2}} \check{\varphi_{\#}}(y) y^{t} \frac{d y}{y}
$$

also holds for $t \in i \mathbb{R}$ ! More trivially, we get the formula

$$
\left\langle E_{\varphi}, \Lambda_{G}^{P} E_{-t}\right\rangle_{\Gamma \backslash \mathbb{H}}=\int_{T}^{\infty} y^{-\frac{1}{2}} \check{\varphi_{\#}}(y) y^{-t} \frac{d y}{y}+c(-t) \int_{T}^{\infty} y^{-\frac{1}{2}} \check{\varphi_{\#}}(y) y^{t} \frac{d y}{y}
$$

for $t \in i \mathbb{R}$ since

$$
\Lambda_{G}^{P} E_{-t}=\sum_{\gamma \in P \cap \Gamma \backslash \Gamma} \chi_{(T, \infty)}\left(E_{-t}\right)_{0}(\operatorname{Im} \gamma z)
$$

has a finite number of nonzero summands. By combining (4.4) and (4.5) for $t \in i \mathbb{R}$, we get

$$
\begin{aligned}
\left\langle E_{\varphi}, E_{-t}\right\rangle_{\Gamma \backslash \mathbb{H}} & =\left\langle E_{\varphi}, \Lambda_{G}^{G} E_{-t}+\Lambda_{G}^{P} E_{-t}\right\rangle_{\Gamma \backslash \mathbb{H}} \\
& =\left\langle E_{\varphi}, \Lambda_{G}^{G} E_{-t}\right\rangle_{\Gamma \backslash \mathbb{H}}+\left\langle E_{\varphi}, \Lambda_{G}^{P} E_{-t}\right\rangle_{\Gamma \backslash \mathbb{H}} \\
& =\int_{0}^{T} y^{-\frac{1}{2}} \check{\varphi_{\#}}(y) y^{-t} \frac{d y}{y}+\int_{T}^{\infty} y^{-\frac{1}{2}} \check{\varphi_{\#}}(y) y^{-t} \frac{d y}{y} \\
& =\int_{0}^{\infty} y^{-\frac{1}{2}} \check{\varphi_{\#}}(y) y^{-t} \frac{d y}{y} \\
& =\varphi_{\#}(t) .
\end{aligned}
$$

4.1. Plancherel formula. We now show how the Fourier inversion formula yields the Plancherel formula.

Theorem 4.1 (Plancherel formula). Let $\varphi$ and $\psi$ be smooth with compact support on $i \mathbb{R}$. Then

$$
\left\langle E_{\varphi}, E_{\psi}\right\rangle_{\Gamma \backslash \mathbb{H}}=\frac{1}{2}\left\langle\varphi_{\#}, \psi_{\#}\right\rangle_{i \mathbb{R}}
$$


Proof. By linearity in the first variable of the Hermitian Petersson inner product, we have

$$
\left\langle E_{\varphi}, E_{\psi}\right\rangle_{\Gamma \backslash \mathbb{H}}=\frac{1}{2 \pi i} \int_{-i \infty}^{i \infty} \varphi(t)\left\langle E_{t}, E_{\psi}\right\rangle_{\Gamma \backslash \mathbb{H}} d t=\left\langle\varphi, \psi_{\#}\right\rangle_{i \mathbb{R}}
$$

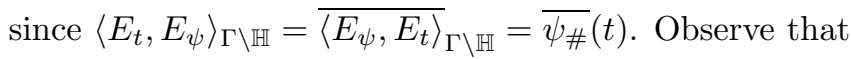

$$
\int_{-i \infty}^{i \infty}[\varphi(t) \overline{c(-t) \psi(-t)}] d t=\int_{-i \infty}^{i \infty}[\varphi(-t) \overline{c(t) \psi(t)}] d t=\int_{-i \infty}^{i \infty}[c(-t) \varphi(-t) \overline{\psi(t)}] d t
$$

and

$$
\int_{-i \infty}^{i \infty} \varphi(t) \overline{\psi(t)} d t=\int_{-i \infty}^{i \infty} \varphi(-t) \overline{\psi(-t)} d t=\int_{-i \infty}^{i \infty} c(-t) \varphi(-t) \overline{c(-t) \psi(-t)} d t
$$

to conclude

$$
\begin{aligned}
2\left\langle\varphi, \psi_{\#}\right\rangle_{i \mathbb{R}} & =2\langle\varphi, \psi\rangle_{i \mathbb{R}}+2\langle\varphi, c(-t) \psi(-t)\rangle_{i \mathbb{R}} \\
& =2\langle\varphi, \psi\rangle_{i \mathbb{R}}+\frac{1}{2 \pi i} \int_{-i \infty}^{i \infty}[\varphi(t) \overline{c(-t) \psi(-t)}+c(-t) \varphi(-t) \overline{\psi(t)}] d t \\
& =\frac{1}{2 \pi i} \int_{-i \infty}^{i \infty}[\varphi(t)+c(-t) \varphi(-t)][\overline{\psi(t)+c(-t) \psi(-t)}] d t \\
& =\left\langle\varphi_{\#}, \psi_{\#}\right\rangle_{i \mathbb{R}} .
\end{aligned}
$$

5. Other proofs of the Plancherel formula

5.1. Langlands' proof. In [6], Langlands starts out with the identity

$$
\left\langle E_{\varphi}, E_{\psi}\right\rangle_{\Gamma \backslash \mathbb{H}}=\frac{1}{2 \pi i} \int_{\operatorname{Re}} \varphi(t) \bar{\psi}(-\bar{t})+c(t) \varphi(t) \bar{\psi}(\bar{t}) d t
$$

for $\sigma>1$. Here, we use the usual Hermitian Petersson inner product. By the residue theorem, he shifts the contour of integration to the line Re $t=0$ and recalls that for such $t, \overline{c(t)}=c(\bar{t})$ and $\bar{t}=-t$ to get the following expression for $\left\langle E_{\varphi}, E_{\psi}\right\rangle_{\Gamma \backslash \mathbb{H}}$ :

$$
\frac{1}{2 \pi i} \int_{-i \infty}^{i \infty} \varphi(t) \bar{\psi}(t)+\overline{c(-t)} \varphi(t) \bar{\psi}(-t) d t+\frac{1}{\xi(2)} \varphi\left(\frac{1}{2}\right) \bar{\psi}\left(\frac{1}{2}\right)
$$

Factoring out $\varphi(t)$ and noticing that $\varphi$ and $\psi$ are supported on the imaginary axis, we see that (5.1) equals

$$
\frac{1}{2 \pi i} \int_{-i \infty}^{i \infty} \varphi(t) \overline{\psi_{\#}}(t) d t
$$

which proves the Plancherel formula.

5.2. Lapid's proof. In [7, Lapid proves there exists a $\delta>0$ such that

$$
\begin{aligned}
& \left\langle\Lambda_{G}^{G} E_{s}, \Lambda_{G}^{G} E_{t}\right\rangle_{\Gamma \backslash \mathbb{H}} \\
& \quad=\frac{1}{2}\left[\frac{T^{s-t}}{s-t}+c(s) \overline{c(t)} \frac{T^{-s+t}}{-s+t}+c(s) \frac{T^{-(s+t)}}{-(s+t)}+\overline{c(t)} \frac{T^{s+t}}{s+t}\right]+O\left(e^{-\delta T}\right)
\end{aligned}
$$

for all $T>0$ sufficiently large and all $(s, t)$ in $i \mathbb{R} \times i \mathbb{R}$. The constant can be chosen uniformly in $(s, t)$ over compact subsets. The following limit

$$
\lim _{T \rightarrow \infty} \frac{1}{2 \pi i} \int_{-i \infty}^{i \infty} \varphi(s)\left[\frac{1}{2}\left[\frac{T^{s-t}}{s-t}+c(s) \overline{c(t)} \frac{T^{-s+t}}{-s+t}+c(s) \frac{T^{-(s+t)}}{-(s+t)}+\overline{c(t)} \frac{T^{s+t}}{s+t}\right]\right] d s
$$


exists and equals

$$
\frac{1}{2}[\varphi(t)+c(t) \overline{c(t)} \varphi(t)+c(-t) \varphi(-t)+\overline{c(t)} \varphi(-t)]
$$

which simplifies to $\varphi_{\#}(t)$. Hence,

$$
\begin{aligned}
\left\langle E_{\varphi}, E_{\psi}\right\rangle_{\Gamma \backslash \mathbb{H}} & =\lim _{T \rightarrow \infty}\left\langle\Lambda_{G}^{G} E_{\varphi}, \Lambda_{G}^{G} E_{\psi}\right\rangle_{\Gamma \backslash \mathbb{H}} \\
& =\frac{1}{2 \pi i} \int_{-i \infty}^{i \infty}\left[\lim _{T \rightarrow \infty} \frac{1}{2 \pi i} \int_{-i \infty}^{i \infty} \varphi(s)\left\langle\Lambda_{G}^{G} E_{s}, \Lambda_{G}^{G} E_{t}\right\rangle d s\right] \overline{\psi(t)} d t \\
& =\frac{1}{2 \pi i} \int_{-i \infty}^{i \infty} \varphi_{\#}(t) \overline{\psi(t)} d t .
\end{aligned}
$$

By symmetry and interchanging the roles of $\varphi$ and $\psi$ in the proof of Theorem 4.1 ,

$$
2\left\langle\varphi_{\#}, \psi\right\rangle_{i \mathbb{R}}=\left\langle\varphi_{\#}, \psi_{\#}\right\rangle_{i \mathbb{R}} .
$$

5.3. Casselman's proof. Casselman's proof in 3 differs from our new proof only in the formula for

$$
\left\langle E_{\varphi}, \Lambda_{G}^{G} E_{-t}\right\rangle_{\Gamma \backslash \mathbb{H} \cdot}
$$

By sesquilinearity of the inner product as well as the idempotence and self-adjointness of the truncation operator, the inner product (5.3) equals

$$
\frac{1}{2 \pi i} \int_{-i \infty}^{i \infty} \varphi(s)\left\langle\Lambda_{G}^{G} E_{s}, \Lambda_{G}^{G} E_{-t}\right\rangle_{\Gamma \backslash \mathbb{H}} d s .
$$

By using the Maass-Selberg formula, (5.4) eventually becomes a convolution of a test function and a distribution:

$$
\left(\varphi_{\#} * P V\left(\frac{T^{-s}}{-s}\right)\right)(t)+c(-t)\left(\varphi_{\#} * P V\left(\frac{T^{-s}}{-s}\right)\right)(-t) .
$$

The inverse Mellin transform of the distribution $P V\left(\frac{T^{-s}}{-s}\right)$ is the function

$$
\operatorname{sgn}_{T}(y)=\left\{\begin{array}{l}
-\frac{1}{2} \text { if } y>T, \\
\frac{1}{2} \text { otherwise. }
\end{array}\right.
$$

Taking the inverse Mellin transform of (5.5) followed by the Mellin transform of the result, we get for $t \in i \mathbb{R}$ :

$$
\left\langle\Lambda_{G}^{G} E_{\varphi}, \Lambda_{G}^{G} E_{-t}\right\rangle_{\Gamma \backslash \mathbb{H}}=\int_{0}^{\infty} y^{-\frac{1}{2}} \check{\varphi_{\#}}(y) \operatorname{sgn}_{T}(y)\left[y^{-t}+c(-t) y^{t}\right] \frac{d y}{y} .
$$

As in the new proof, for $t \in i \mathbb{R}$ we get

$$
\left\langle\Lambda_{G}^{P} E_{\varphi}, \Lambda_{G}^{P} E_{-t}\right\rangle_{\Gamma \backslash \mathbb{H}}=\int_{0}^{\infty} y^{-\frac{1}{2}} \check{\varphi_{\#}}(y) \chi_{(T, \infty)}(y)\left[y^{-t}+c(-t) y^{t}\right] \frac{d y}{y} .
$$

Since $\operatorname{sgn}_{T}+\chi_{(T, \infty)}=\frac{1}{2}$,

$$
\begin{aligned}
\left\langle E_{\varphi}, E_{-t}\right\rangle_{\Gamma \backslash \mathbb{H}} & =\frac{1}{2}\left[\int_{0}^{\infty} y^{-\frac{1}{2}} \check{\varphi_{\#}}(y) y^{-t} \frac{d y}{y}+c(-t) \int_{0}^{\infty} y^{-\frac{1}{2}} \check{\# \#}(y) y^{t} \frac{d y}{y}\right] \\
& =\frac{1}{2}\left[\varphi_{\#}(t)+c(-t) \varphi_{\#}(-t)\right] \\
& =\varphi_{\#}(t) .
\end{aligned}
$$




\section{ACKNOWLEDGMENTS}

The author would like to thank Jonathan Rogawski for suggesting this problem and for his constant help and guidance. Bill Casselman's proof provided the inspiration for this idea. Many thanks to Erez Lapid and Bill Casselman for many helpful discussions. Finally, the author extends his gratitude to the referee for a careful reading of this article and for several useful suggestions.

\section{REFERENCES}

1. James G. Arthur, A (very brief) history of the trace formula, A note on http://www. claymath.org/cw/arthur/pdf/HistoryTraceFormula.pdf, 2007.

2. Armand Borel, Automorphic forms on $\mathrm{SL}_{2}(\mathbf{R})$, Cambridge Tracts in Mathematics, vol. 130, Cambridge University Press, Cambridge, 1997. MR.1482800 (98j:11028)

3. William A. Casselman, On the plancherel measure for the continuous spectrum of the modular group, Automorphic forms, automorphic representations, and arithmetic (Fort Worth, TX, 1996), Proc. Sympos. Pure Math., vol. 66, Amer. Math. Soc., Providence, RI, 1999, pp. 1925. MR 1703754 (2000c:11003)

4. Gerald B. Folland, Real analysis, second ed., Pure and Applied Mathematics (New York), John Wiley \& Sons Inc., New York, 1999, Modern techniques and their applications, A WileyInterscience Publication. MR 1681462 (2000c:00001)

5. Tomio Kubota, Elementary theory of Eisenstein series, Kodansha Ltd., Tokyo, 1973. MR 0429749 (55:2759)

6. R. P. Langlands, Eisenstein series, Algebraic Groups and Discontinuous Subgroups (Proc. Sympos. Pure Math., Boulder, Colo., 1965), Amer. Math. Soc., Providence, R.I., 1966, pp. 235-252. MR0249539(40:2784)

7. Erez Lapid, On Arthur's asymptotic inner product formula of truncated Eisenstein series, to appear in Clay Mathematics Proceedings.

8. Hans Maass, Über eine neue Art von nichtanalytischen automorphen Funktionen und die Bestimmung Dirichletscher Reihen durch Funktionalgleichungen, Math. Ann. 121 (1949), 141-183. MR0031519(11,163c)

9. Colette Mœglin and Jean-Loup Waldspurger, Décomposition spectrale et séries d'Eisenstein, Progress in Mathematics, vol. 113, Birkhäuser Verlag, Basel, 1994, Une paraphrase de l'Écriture. [A paraphrase of Scripture]. MR1261867 (95d:11067)

10. W. Roelcke, Analytische Fortsetzung der Eisensteinreihen zu den parabolischen Spitzen von Grenzkreisgruppen erster Art, Math. Ann. 132 (1956), 121-129. MR0082562 (18:571b)

11. Walter Rudin, Functional analysis, McGraw-Hill Book Co., New York, 1973, McGraw-Hill Series in Higher Mathematics. MR0365062 (51:1315)

12. Atle Selberg, Harmonic analysis and discontinuous groups in weakly symmetric Riemannian spaces with applications to Dirichlet series, J. Indian Math. Soc. (N.S.) 20 (1956), 47-87. MR0088511 (19:531g)

13. , Discontinuous groups and harmonic analysis, Proc. Internat. Congr. Mathematicians (Stockholm, 1962), Inst. Mittag-Leffler, Djursholm, 1963, pp. 177-189. MR0176097 (31:372)

14. V. S. Varadarajan, An introduction to harmonic analysis on semisimple Lie groups, Cambridge Studies in Advanced Mathematics, vol. 16, Cambridge University Press, Cambridge, 1999, Corrected reprint of the 1989 original. MR:1725738 (2000m:22011) 01610

Department of Mathematics, College of the Holy Cross, Worcester, Massachusetts

E-mail address: kouellet@holycross.edu 